\title{
Clapotis partiel
}

\section{Partial clapotis}

\author{
PAR C. CARRY
}

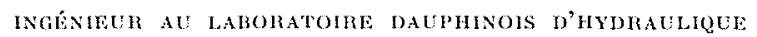

\begin{abstract}
Des équations générales anl second ordre des moumements résultant de la superposition de plusieurs trains de houles, établies par M. Bress.. l'antenr tire les équations générales du clapotis partiel.

A ces equations sont joints des abaques permettant, a partir de mesures expérimentales, de connatre le coefficient de réflexion d'un ouvrage et les caracteristiques de la houle incidente.
\end{abstract}

\begin{abstract}
From second order general equations, established by $\mathrm{Mr}$. Biessi, of movements resulting from the superposition of several wave trains, the author evolves general equations of the partial clapotis. Graphs are attached to these equations enabling, afler the experimental measurements, knowledge of the reffection coefficient of a structure and the characterislics of the incidental wave.
\end{abstract}

Dans un article précédent, M. Bissfe * a établi les équations générales au second ordre des mouvements résultant de la superposition de plusieurs trains de houles.

Nous nous proposons d'appliquer ces formules au cas particulier du Clapotis Partiel qui résulte de la rencontre de deux houles régulières d'amplitudes différentes mais de même fréquence. Ce type de mouvement se produit en particulier devant les modèles des ouvrages partiellement réfléchissants attaqués de front par une houle régulière. Son étude est donc particulièrement intéressante pour l'interprétation des expériences destinées à déterminer le coefficient de réflexion de tels ouvrages. Il est entendu, bien que nous ne le répétions pas systématiquement, que tous les calculs qui suivent sont poussés au second ordre d'approximation seulement, les termes du troisième ordre étant considérés comme négligeables.

Nous rappelons tout d'abord les formules de M. BIFsec (dépliant ci-joint) où les notations utilisces sont les suivantes:

$$
\begin{aligned}
\mathrm{O} x, \mathrm{O} x_{0} & =\text { axes de coordonnées horizontaux dirigés de gauche à droite, confondus } \\
\text { avec le niveau de repos du liquide } & \\
\mathrm{O} y, \mathrm{O} y_{0} & =\text { axes de coordonnées verticaux descendants. } \\
x, y & =\text { coordonnées instantanées des particules en mouvement. } \\
x_{0}, y_{0} & =\text { coordonnées "initiales } \text { ou au repos des particules. } \\
2 a_{i} & =\text { amplitude de la houle composante } \mathrm{n} \text { " } i \text {. } \\
\mathrm{L}_{i} & =\text { longueur d'onde de la houle composante } \mathrm{n} \text { " } i .
\end{aligned}
$$

* Voir la Honille blanche, n*3,1952. 


\section{Équations générales au second ordre de la houle irrégulière}

General 2nd order equations of irregular waves

D'APRES F. BIESTEL

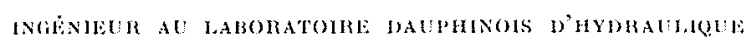

(La Houlle Blanche, n" 3, 1952, p. 372.)

(Complement is l'article publie dans le present muméro, $p$. 482.) 


\section{EQUATIONS GENERALES}

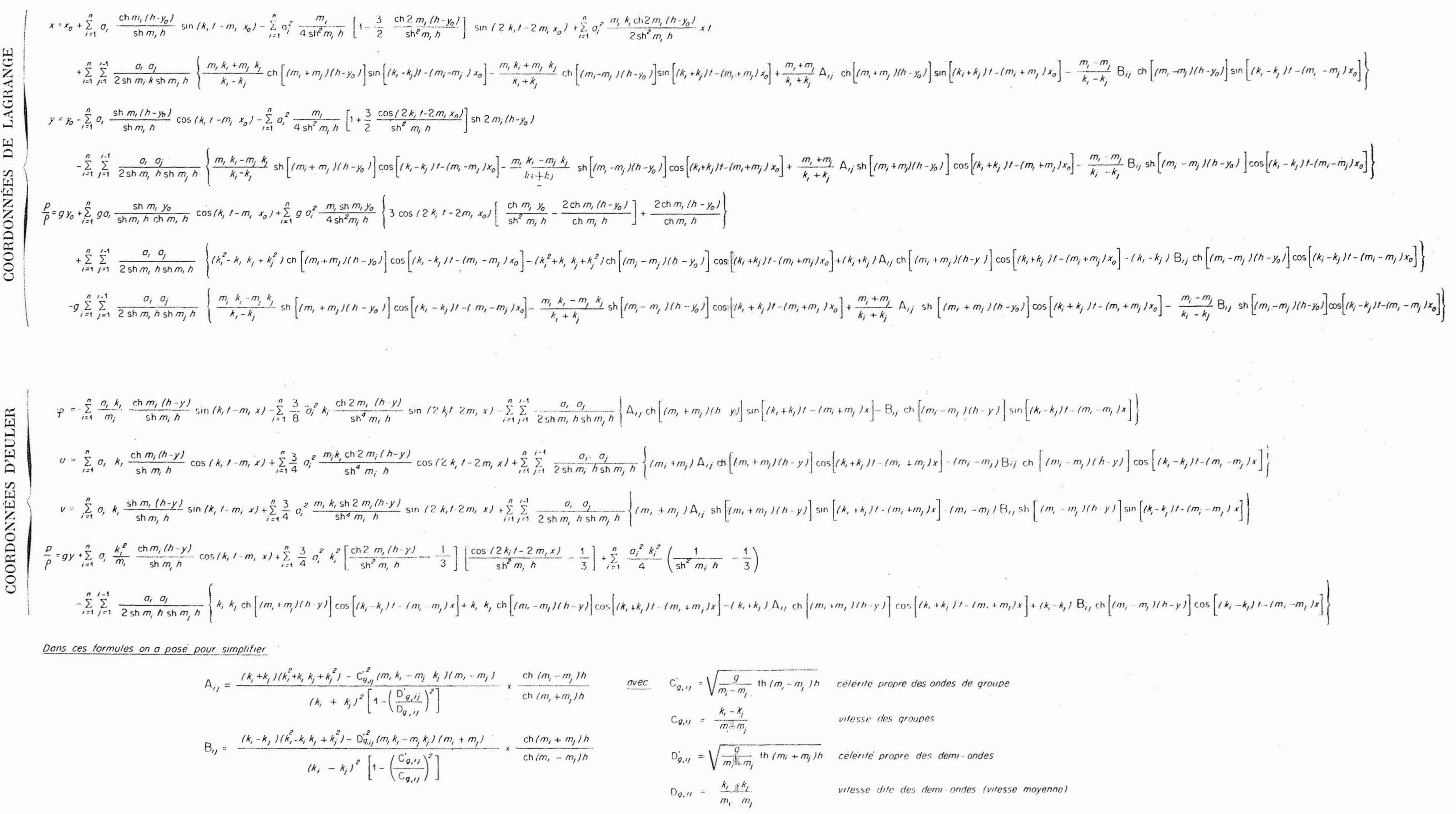




\section{BATTEMENT EN PROFONDEUR QUELCONQUE}

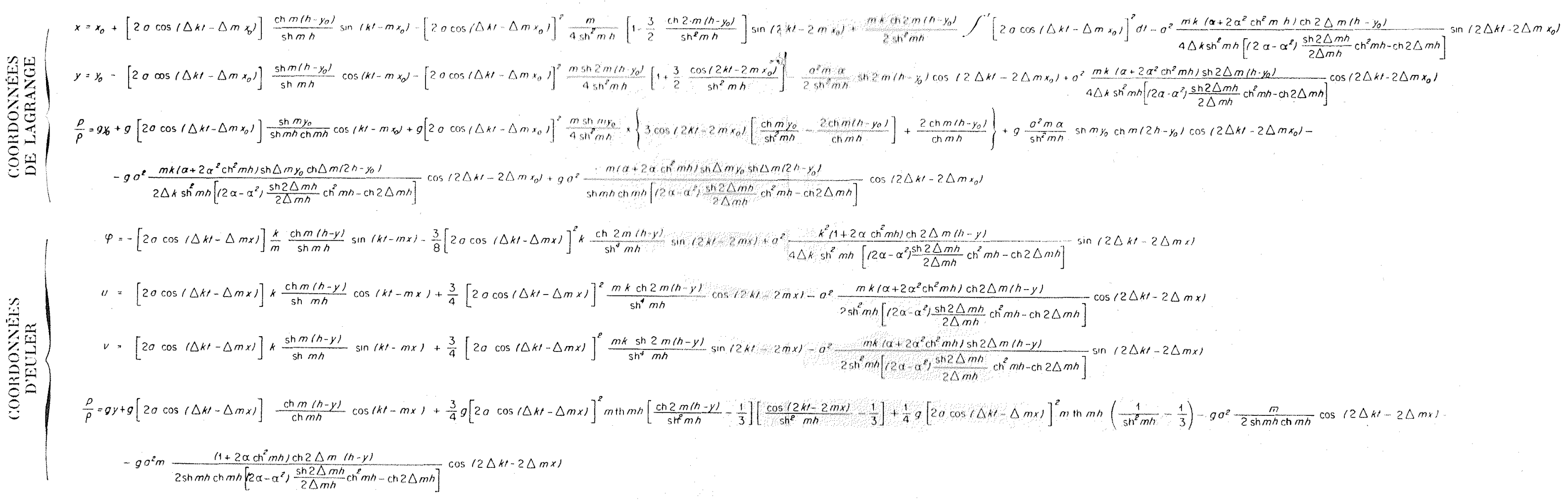

\section{CLAPOTIS IMPARFAIT EN PROFONDEUR QUELCONQUE}

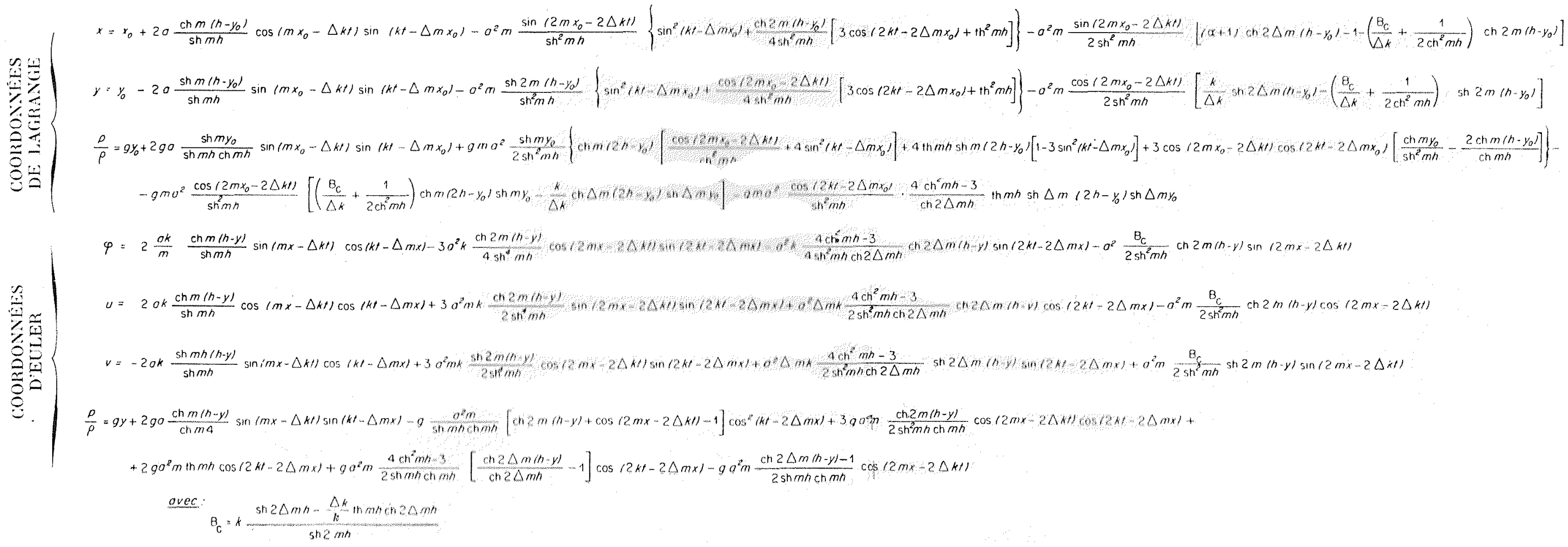




\section{BATTEMENT EN PROFONDEUR INFINIE}

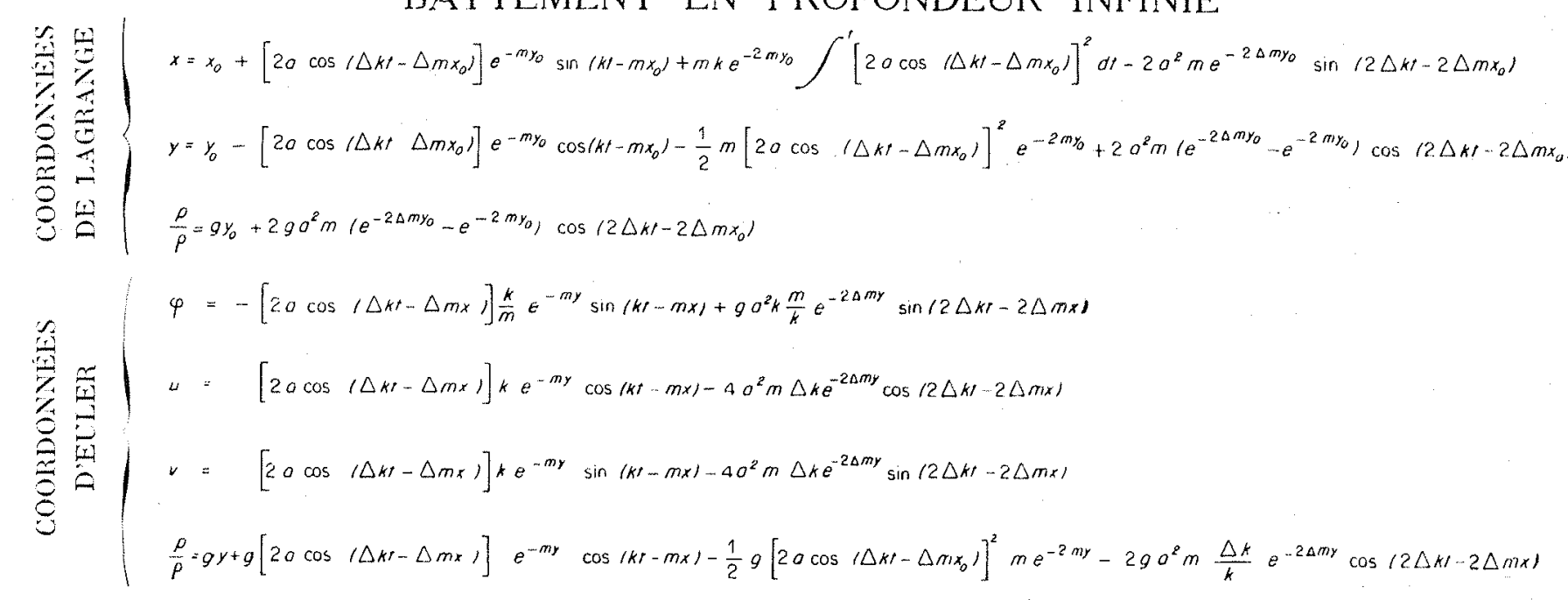

HOULE REGULIERE EN PROFONDEUR QUELCONQUE

$$
\text { (Formules données à titre de comparaison.) }
$$

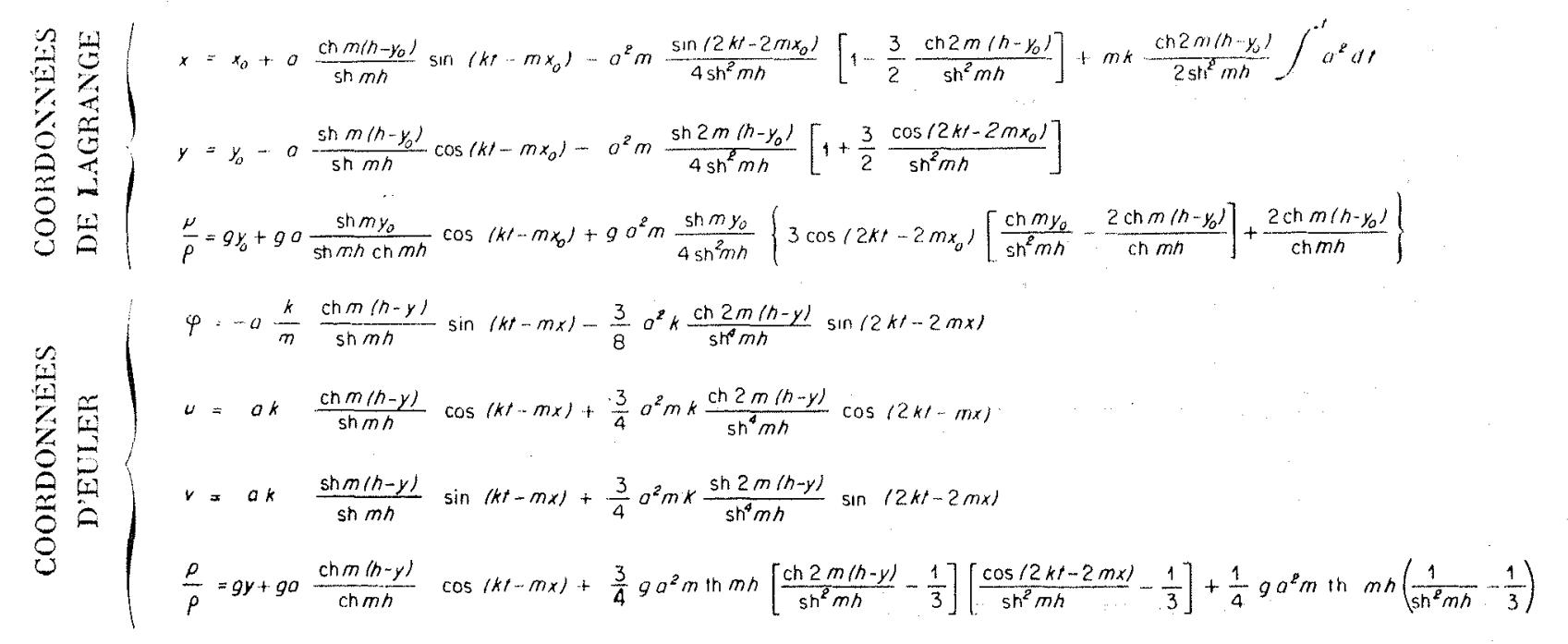

HOULE REGULIERE EN PROFONDEUR INFINIE. (Formules données à titre de comparaison.)

$$
\begin{aligned}
& x=x_{0}+a e^{-m x_{0}} \sin \left(k t-m x_{0}\right)+m k e^{-2 m x_{0}} \int a_{a^{2} d t}^{\prime} \\
& y=x_{0}-a e^{-m x_{0}} \cos \left(k t-m x_{x_{a}}\right)-\frac{a^{2} m}{2} e^{-2 m y_{0}} \\
& \frac{\rho}{\rho}=9 \%_{0} \\
& \varphi=0 \frac{k}{m} e^{-a t y} \sin (k t-m r) \\
& u=O x e^{-m r} \cos (k t-m x) \\
& v=0 x e^{-m y} \sin (k t-m x) \\
& \frac{\rho}{\bar{p}}=g y+g a e^{-m y} \cos \left(k t-m_{x}\right)-\frac{1}{2} g a^{2} m e^{-2 m y}
\end{aligned}
$$

CLAPOTIS IMPARFAIT EN PROFONDEUR INFINIE
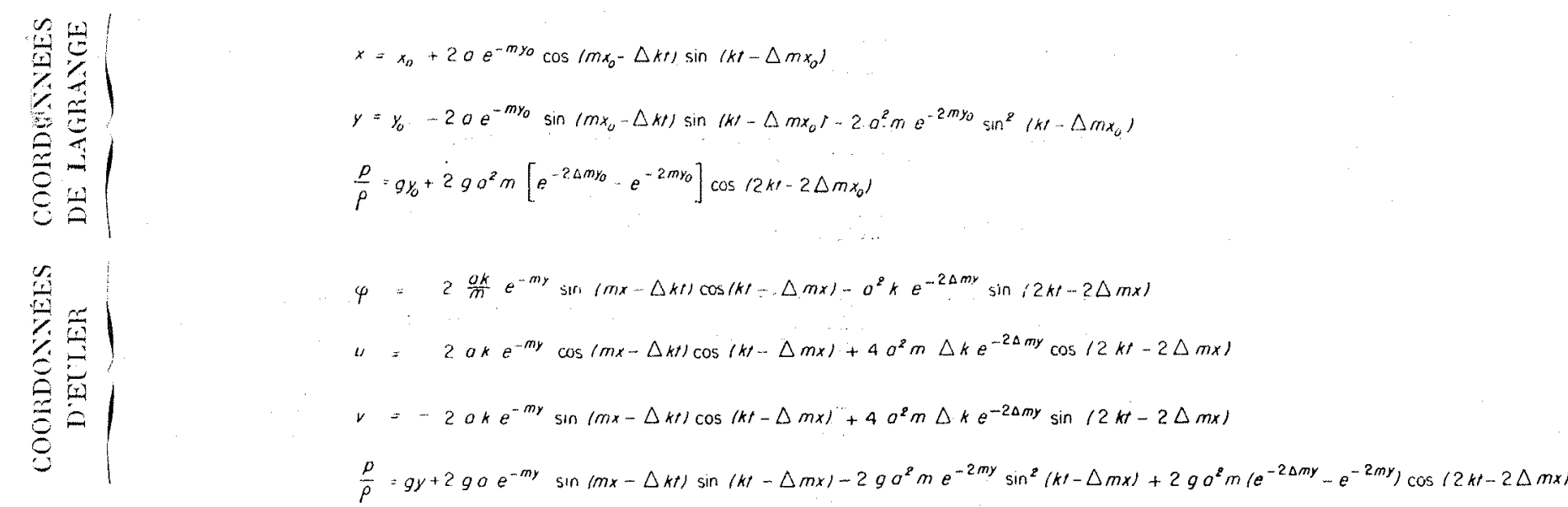

CLAPOTIS REGULIER EN PPOFONDEUR QUELCONQUE (Formules données à titre de comparaison.)

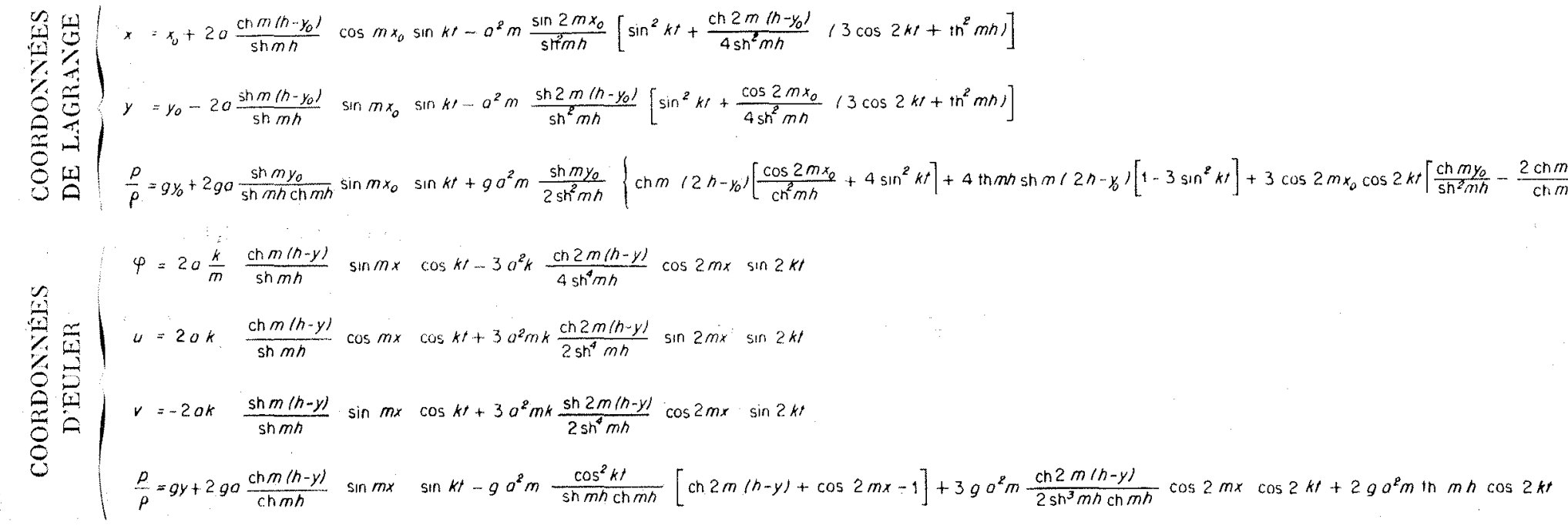

CLAPOTIS REGULIER EN PROFONDEUR INFINIE. (Formules données à titre de comparaison.)

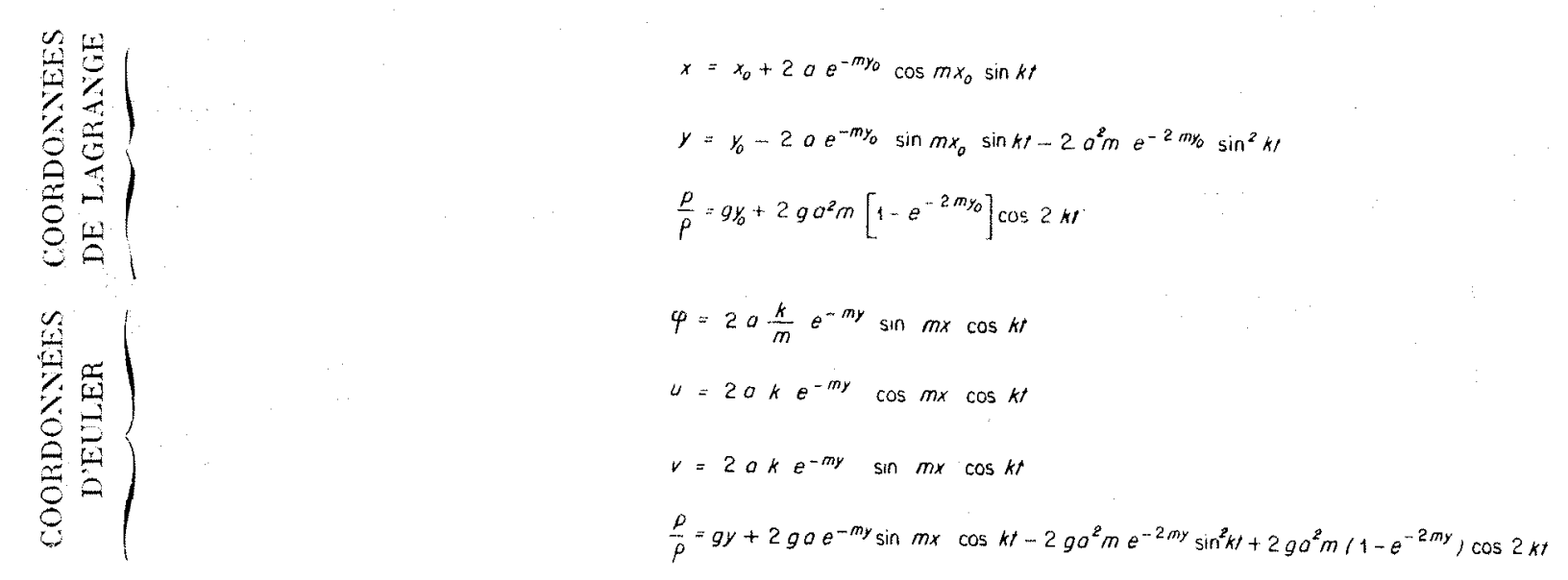


$\mathrm{T}_{i}=$ période de la houle composante $\mathrm{n}$ " $i$.

$m_{i}=\frac{2 \pi}{\mathrm{L}_{t}}$ «nombre d'onde $»$.

$k_{i}=\frac{2 \pi}{\mathrm{T}}$ fréquences angulaires.

$g=$ accélération de la pesanteur.

$h=$ profondeur moyenne de l'eau.

$m_{i}, k_{i}$ et $h$ sont liés par la relation classique $k_{i}^{2}=m_{i} g$ th $m_{i} h$.

Nous considérerons alors les équations relatices à deux houles composantes d'indices 1 et 2 et nous poserons :

$$
m_{1}=-m_{2}=m \quad k_{1}=k_{2}=k
$$

Nous prendrons comme amplitude $a_{1}$ et $-a_{2}{ }^{* *}$.

Certains coefficients des formules générales prennent une valeur indéterminée par suite des relations ci-dessus. Il est facile de lever cette indétermination par Ie procédé classique du passage à la limite. On obtient ainsi en particulier :

$$
\begin{gathered}
\mathrm{A}_{12}=\frac{k}{2}[2 \operatorname{ch} 2 m h-1]=\frac{m_{1}+m_{2}}{k_{1}-k_{2}}=\frac{4 k \mathrm{ch}^{2} m h}{g(2 m h+\operatorname{sh} 2 m h)} \\
\frac{B_{12}}{k_{1}-k_{2}}=\frac{1}{4}\left[\mathrm{th}^{2} m h+\frac{6 m h-\operatorname{sh} 2 m h}{2 m h+\operatorname{sh} 2 m h}\right]
\end{gathered}
$$

Nous sommes maintenant en mesure d'écrire les formules du clapotis.

\section{CLAPOTIS PARTIEL}

\section{En coordonnées de Lagrange :}

$$
\begin{aligned}
& x=x_{t_{1}}+\frac{\operatorname{ch} m\left(h-y_{0}\right)}{\operatorname{sh} m h}\left[\left(a_{1}+a_{2}\right) \sin k t \cos m x_{0}-\left(a_{1}-a_{2}\right) \cos k t \sin m x_{01}\right] \\
& +\frac{m}{4 \operatorname{sh}^{2} m h}\left[1-\frac{3 \operatorname{ch} 2 m\left(h-y_{0}\right)}{2 \operatorname{sh}^{2} m h}\right]\left[\left(a_{2}{ }^{2}-a_{1}{ }^{2}\right) \sin 2 k t \cos 2 m x_{0}+\left(a_{1}{ }^{2}+a_{2}{ }^{2}\right) \cos 2 k t \sin 2 m x_{0}\right] \\
& +\frac{m a_{0} a_{1} \sin 2 m x_{0}}{2 \operatorname{sh}^{2} m h}\left[-\frac{3 \operatorname{sh} 2 m h+2 m h}{\operatorname{sh} 2 m h+2 m h}+\frac{\operatorname{ch} 2 m\left(h-y_{0}\right)}{2}\left(\operatorname{th}^{2} m h+\frac{6 m h-\operatorname{sh} 2 m h}{2 m h+\operatorname{sh} 2 m h}\right)\right] \\
& y=y_{0}-\frac{\operatorname{sh} m\left(h-y_{0}\right)}{\operatorname{sh} m h}\left[\left(a_{1}-a_{2}\right) \cos m x_{0} \cos k t+\left(a_{1}+a_{2}\right) \sin m x_{0} \sin k t\right] \\
& -\frac{m \operatorname{sh} 2 m\left(h-y_{0}\right)}{4 \operatorname{sh}^{2} m h}\left[a_{1}^{2}+a_{2}^{2}+\frac{3}{2} \frac{\left(a_{1}^{2}+a_{2}^{2}\right) \cos 2 k t \cos 2 m x_{0}+\left(a_{1}^{2}-a_{0}^{2}\right) \sin 2 m x_{0} \sin 2 k t}{\operatorname{sh}^{2} m h}\right] \\
& +\frac{m a_{2} a_{1}}{2 \operatorname{sh}^{2} m h}\left\{\operatorname{sh} 2 m\left(h-y_{0}\right)\left[\cos 2 h t+\frac{1}{2}\left(t h^{2} m h+\frac{6 m h-\operatorname{sh} 2 m h}{2 m h+\operatorname{sh} 2 m h}\right) \cos 2 m x_{0}\right]\right. \\
& -\frac{4 m\left(h-\underline{y}_{0}\right) \operatorname{sh}^{2} m h}{2 m h+\operatorname{sh} 2 m h} \cos 2 m x_{0}
\end{aligned}
$$

* Nous avous pris - a.g pour obtenir des formules comparables à celles de M. Mache. 
$\frac{p}{\rho g}=y_{0}+\frac{2 \operatorname{sh} m y_{0}}{\operatorname{sh}^{2} m h}\left[\left(a_{1}-a_{2}\right) \cos k t \cos m x_{0}+\left(a_{1}+a_{2}\right) \sin k t \sin m x_{0}\right]$

$+\frac{3 m \operatorname{sh} m y_{0}}{4 \operatorname{sh}^{2} m h}\left[\frac{\operatorname{ch} m y_{0}}{\operatorname{sh}^{2} m h}-\frac{2 \operatorname{ch} m\left(h-y_{0}\right)}{\operatorname{ch} m h}\right]\left[\left(a_{1}{ }^{2}+a_{2}{ }^{2}\right) \cos 2 k t \cos 2 m x_{0}+\left(a_{1}{ }^{2}-a_{2}{ }^{2}\right) \sin 2 k t \sin 2 m x_{0}\right]$

$+\frac{m\left(a_{1}^{2}+a_{2}^{2}\right) \operatorname{sh} m y_{0} \operatorname{ch} m\left(h-y_{0}\right)}{2 \operatorname{ch} m h \operatorname{sh}^{2} m h}$

$+\frac{m a_{1} a_{2}}{2 \operatorname{sh}^{2} m h}\left(\left[\operatorname{th} m h\left(2 \operatorname{ch} 2 m h-1-3 \operatorname{ch} 2 m\left(h-y_{0}\right)\right)+\operatorname{sh} 2 m\left(h-y_{0}\right)\right] \cos 2 k t\right.$

$\left.+\left[\operatorname{th} m h-\frac{4 m\left(h-y_{0}\right) \operatorname{sh} 2 m h}{2 m h+\operatorname{sh} 2 m h}+\frac{\operatorname{sh} 2 m\left(h-y_{0}\right)}{2} \operatorname{th}^{2} m h+\frac{(m h-\operatorname{sh} 2 m h)}{2 m h+\operatorname{sh} 2 m h}\right] \cos 2 m x_{0}\right)$

\section{En coordonnées d'Euler :}

$$
\begin{aligned}
& \left.\varphi=\frac{k}{m}\right\} \frac{\operatorname{ch} m(h-y)}{\operatorname{sh} m h}\left[\left(a_{1}+a_{2}\right) \sin k t \cos m x-\left(a_{1}-a_{2}\right) \cos l_{i} t \cos m x\right] \\
& -\frac{3 m \operatorname{ch} 2 m(h-y)}{8 \operatorname{sh}^{4} m h}\left[\left(a_{1}^{2}+a_{2}^{2}\right) \sin 2 k t \cos 2 m x+\left(a_{2}{ }^{2}-a_{1}{ }^{2}\right) \cos 2 k t \sin 2 m x\right] \\
& -\frac{m a_{1} a_{2}(2 \operatorname{ch} 2 m h-1)}{4 \operatorname{sh}^{2} m h} \sin 2 k t \\
& \mathrm{U}=k\left\{\frac{\operatorname{ch} m(h-y)}{\operatorname{sh} m h}\left[\left(a_{1}+a_{2}\right) \cos k \mathrm{t} \cos m x+\left(a_{1}-a_{2}\right) \sin k t \sin m x\right]\right. \\
& \left.+\frac{3}{4} \frac{m \operatorname{ch} 2 m(h-y)}{\operatorname{sh}^{4} m h}\left[\left(a_{1}{ }^{2}-a_{2}{ }^{2}\right) \cos 2 k t \cos 2 m x+\left(a_{1}{ }^{2}+a_{2}{ }^{2}\right) \sin 2 m x \sin 2 k t\right]\right\} \\
& V=k\left\{\frac{\operatorname{sh} m(h-y)}{\operatorname{sh} m h}\left[\left(a_{1}+a_{2}\right) \sin k t \cos m x+\left(a_{2}-a_{1}\right) \sin m x \cos k t\right]\right. \\
& \left.+\frac{3 m \operatorname{sh}^{2} m(h-y)}{4 \operatorname{sh}^{t} m h}\left[\left(a_{1}{ }^{2}+a_{2}{ }^{2}\right) \sin 2 k t \cos 2 m x+\left(a_{2}{ }^{2}-a_{1}{ }^{2}\right) \sin 2 m x \cos 2 k t\right]\right\} \\
& \frac{p}{\rho}=g y+\frac{k^{2}}{m}\left\{\frac{\operatorname{ch} m(h-y)}{\operatorname{sh} m h}\left[\left(a_{1}-a_{2}\right) \cos k t \cos m x+a_{1}+a_{2}\right) \sin k t \sin m x\right] \\
& +\frac{3 m}{4 \operatorname{sh}^{2} m h}\left[\frac{(\ln 2 m(h-y)}{\operatorname{sh}^{2} m h}-\frac{1}{3}\right]\left[\left(a_{1}^{2}+a_{22^{2}}\right) \cos 2 k t \cos 2 m x+\left(a_{1}{ }^{2}-a_{2}{ }^{2}\right) \sin 2 k t \sin 2 m x\right] \\
& +\frac{m\left(a_{1}^{2}+a_{2}^{2}\right)}{4 \operatorname{sh}^{2} m h}[1-\operatorname{ch} 2 m(h-y)] \\
& \left.-\frac{m a_{1} a_{2}}{2 \operatorname{sh}^{2} m h}\lceil\cos 2 m x+[\operatorname{ch} 2 m(h-y)-2 \operatorname{ch} 2 m h+1] \cos 2 k t]\right\}
\end{aligned}
$$




\section{CLAPOTIS PARFAIT}

En faisant $a_{1}=a_{2}=a$ dans les formules précédentes, nous obtenons celles du clapotis parfait.

\section{En coordonnées de Lagrange :}

$$
\begin{aligned}
& \left.x=x_{0}+2 a \frac{\operatorname{ch} m\left(h-y_{0}\right)}{\operatorname{sh} m h} \sin k t \cos m x_{0}+\frac{m a^{2}}{2 \operatorname{sh}^{2} m h}\right)\left(1-\frac{3}{2} \frac{\operatorname{ch} 2 m\left(h-y_{0}\right)}{\operatorname{sh}^{2} m h}\right) \cos 2 k t \sin 2 m x_{0} \\
& +\left[-\frac{3 \operatorname{sh} 2 m h+2 m h}{\operatorname{sh} 2 m h+2 m h}+\frac{\operatorname{ch}^{2} m\left(h-y_{0}\right)}{2}\left(\frac{6 m h-\operatorname{sh} 2 m h}{2 m h+\operatorname{sh} 2 m h}+t^{2} m h\right)\right] \sin 2 m x_{0} ! \\
& y=y_{0}-2 a \frac{\operatorname{sh} m\left(h-y_{0}\right)}{\operatorname{sh} m h} \sin m x_{0} \sin k t-\frac{m a^{2}}{2 \operatorname{sh}^{2} m h} \mid \operatorname{sh} 2 m\left(h-y_{0}\right) \\
& \left\lceil 1+\frac{3 \cos 2 k t \cos 2 m x_{11}}{2 \operatorname{sh}^{2} m h}-\cos 2 k t-\frac{1}{2}\left(t h^{2} m h+\frac{6 m h-\operatorname{sh} 2 m h}{2 m h+\operatorname{sh} 2 m h}\right) \cos 2 m x_{0} \mid\right. \\
& \left.+\frac{4 m\left(h-y_{0}\right) \operatorname{sh} 2 m h}{2 m h+\operatorname{sh} 2 m h} \cos 2 m x_{0}\right\} \\
& \frac{p}{q g}=y_{0}+\frac{4 a \operatorname{sh} m y_{01}}{\operatorname{sh} m h} \sin k t \sin m x_{11} \\
& \left.+\frac{m a^{2}}{2 \operatorname{sh}^{2} m h}\right\} 3 \operatorname{sh~} m y_{0}\left(\frac{\operatorname{ch} m y_{0}}{\operatorname{sh}^{2} m h}-\frac{2 \operatorname{ch} m\left(h-y_{0}\right)}{\operatorname{ch} m h}\right) \cos 2 k t \cos 2 m x_{0}+\frac{2 \operatorname{sh} m y_{0} \operatorname{ch} m\left(h-y_{0}\right)}{\operatorname{ch} m h} \\
& +\left\lceil\operatorname{th} m h\left(2 \operatorname{ch}^{2} m h-1-3 \operatorname{ch}^{2} m\left(h-y_{0}\right)\right)+\operatorname{sh} 2 m\left(h-y_{0}\right)\right\rceil \cos 2 k t \\
& \left.+\left[\text { th } m h+\frac{\operatorname{sh} 2 m\left(h-y_{0}\right)}{2}\left(t h^{2} m h+\frac{6 m h-\operatorname{sh} 2 m h}{2 m h+\operatorname{sh} 2 m h}\right)\right] \cos 2 m x_{0}\right)
\end{aligned}
$$

\section{En coordonnées d'Euler :}

$$
\begin{aligned}
q & =\frac{k}{m}\left\{+2 a \frac{\operatorname{ch} m(h-y)}{\operatorname{sh} m h} \sin k t \cos m x\right. \\
& \left.-\frac{m a^{2}}{4 \operatorname{sh}^{2} m h}\left[\frac{3 \operatorname{ch} 2 m(h-y)}{\operatorname{sh}^{2} m h} \sin 2 k t \cos 2 m x+(2 \operatorname{ch} 2 m h-1) \sin 2 k t\right]\right\} \\
\mathrm{U} & =k\left[\frac{2 a \operatorname{ch} m(h-y)}{\operatorname{sh} m h} \cos k t \cos m x+\frac{3}{2} \frac{m a^{2} \operatorname{ch} 2 m(h-y)}{\operatorname{sh}^{4} m h} \cos 2 k t \cos 2 m x\right] \\
\mathrm{V} & =k\left[+2 a \frac{\operatorname{sh} m(h-y)}{\operatorname{sh} m h} \sin k t \cos m x+\frac{3 m a^{2} \operatorname{sh}^{4} m(h-y)}{2 \operatorname{sh}^{4} m h} \sin 2 k t \cos 2 m x\right]
\end{aligned}
$$




$$
\begin{aligned}
\frac{p}{\rho} & =g y+\frac{k^{2}}{m}\left\{\frac{2 a \operatorname{ch} m(h-y)}{\operatorname{sh} m h} \sin m x \sin k t\right. \\
+ & \begin{array}{r}
2 a^{2} \\
\operatorname{sh}^{2} m h
\end{array}\left[3\left(\frac{\operatorname{ch} 2 m(h-y)}{\operatorname{sh}^{2} m h}-\frac{1}{3}\right) \cos 2 k t \cos 2 m x+1-\operatorname{ch} 2 m(h-y)-\cos 2 m x\right. \\
& -(\operatorname{ch} 2 m(h-y)-2 \operatorname{ch} 2 m h+1) \cos 2 k t]\}
\end{aligned}
$$

En examinant les formules précédentes, nous constatons qu'elles présentent de légères différences avec celles de M. Mrche, les voici :

pour $x$ :

$$
\frac{m a^{2}}{\operatorname{sh}^{2} m h}\left[\frac{\operatorname{sh} 2 m h-2 m h \operatorname{ch} 2 m\left(h-y_{0}\right)}{2 m h+\operatorname{sh} 2 m h}\right] \sin 2 m x_{0}
$$

pour $y$ :

pour $\frac{n}{p g}$ :

$$
-2 m a^{2}\left\lceil\frac{m h \operatorname{sh} 2 m\left(h-y_{0}\right)-m\left(h-y_{0}\right) \operatorname{sh} 2 m h}{\operatorname{sh}^{2} m h(2 m h+\operatorname{sh} 2 m h)}\right] \cos 2 m x_{0}
$$$$
-4 m a^{2}\left[\frac{m h \operatorname{sh}^{2} m\left(h-y_{0}\right)-m\left(h-y_{0}\right) \operatorname{sh} 2 m h}{\operatorname{sh}^{2} m h(2 m h+\operatorname{sh} 2 m h)}\right.
$$

pour $\varphi$ :

$$
-\frac{k a^{2}}{4 \operatorname{sh}^{2} m h}(2 \operatorname{ch} 2 m h-1) \sin 2 k t
$$

Cette dernière différence est sans importance physique puisqu'elle ne dépend que du temps. Les autres différences, qui s'annulent d'ailleurs pour $y$ et $p$ à la surface et au fond, proviennent de ce que les définitions de $x_{0}$ et $y_{0}$ caractérisant une particule ne sont pas exactement les mêmes chez MM. Bifsel et Miche, quoique on ait au fond $y_{0}=h$ et à la surface $y_{0}=0$ chez tous les deux.

N.B. - Si nous appelons $x_{0} y_{0}$ la place d'une particule au repos chez M. Breses et $x_{1} y_{1}$ la place de la mème particule au repos chez M. Miche, nous avons:

$$
\begin{aligned}
& x_{0}=x_{1}+\frac{m a^{2}\left\lceil\operatorname{sh} 2 m h-2 m h \operatorname{ch} 2 m\left(h-y_{1}\right)\right\rceil}{\operatorname{sh}^{2} m h(2 m h+\operatorname{sh} 2 m h)} \sin 2 m x_{1} \\
& y_{0}=y_{1}-\frac{2 m \alpha^{2}\left[m h \operatorname{sh} 2 m\left(h-y_{1}\right)-m\left(h-y_{1}\right) \operatorname{sh} 2 m h\right\rceil}{\operatorname{sh}^{2} m h(2 m h+\operatorname{sh} 2 m h)} \cos 2 m x_{1}
\end{aligned}
$$

Ces deux systèmes de positions des particules au repos sont également acceptables, car ils satisfont à l'équation de continuité d'un fluide incompressible :

$$
\frac{\partial x_{1}}{x_{0}} \frac{\partial y_{1}}{\partial y_{0}}-\frac{\partial x_{1}}{\partial y_{0}} \frac{\partial y_{1}}{\partial x_{0}}=\frac{\partial x_{2}}{\partial x_{0}} \frac{\partial y_{2}}{\partial y_{0}}-\frac{\partial x_{2}}{\partial y_{0}} \frac{\partial y_{0}}{\partial x_{0}}=1
$$

La divergence entre les deux systèmes de formules n'est donc qu'apparente et ne les empêche pas d'être physiquement équivalents à l'ordre d'approximation considéré. Sous réserves d'études poussées en troisième ordre, il est d'ailleurs impossible de faire un choix rationnel entre ces deux représentations. Tout ce que l'on peut dire est que le système de coordonnées intiales utilisé par M. Miche conduit à des formules un peu plus simples tandis que le système utilisé par M. Breser se racorde d'une façon continue à la famille d'équations plus générale qu'il a établie dans l'article déjà cité.

\section{SURFACE LIBRE}

En faisant $y=\mathbf{0}$ dans les formules précédentes, nous obtenons les équations paramétriques des surfaces libres, $x_{0}$ étant le paramètre. 


\section{Clapotis partiel :}

$$
\begin{aligned}
& x=x_{0}+\frac{1}{\text { th } m h}\left[\left(a_{1}+a_{2}\right) \sin k t \cos m x_{0}-\left(a_{1}-a_{2}\right) \cos k t \sin m x_{0}\right] \\
& --\frac{m(2 \operatorname{ch} 2 m h+1)}{8 \operatorname{sh}^{4} m h}\left[\left(a_{2}^{2}-a_{1}^{2}\right) \sin 2 k t \cos m x_{0}+\left(a_{2}{ }^{2}+a_{1}^{2}\right) \cos 2 k t \sin 2 m x_{0}\right] \\
& +\frac{m a_{1}\left(a_{1} \sin 2 m x_{0}\right.}{4 \operatorname{sh}^{2} m h}\left[\frac{-6 \operatorname{sh} 2 m h-4 m h+\operatorname{ch} 2 m h(6 m h-\operatorname{sh} 2 m h)}{\operatorname{sh} 2 m h+2 m h}+\text { the }^{2} m h \operatorname{ch} 2 m h\right. \\
& \left.y=-\left[\left(a_{1}-a_{2}\right) \cos m x_{0} \cos k t+\left(a_{1}+a_{2}\right) \sin m x_{0} \sin k t\right)\right] \\
& -\frac{m}{2 \operatorname{th} m h}\left[a_{1}^{2}+a_{2}^{2}+\frac{3}{2} \frac{\left(a_{1}^{2}+a_{0}^{2}\right) \cos 2 k t \cos 2 m x_{n}+\left(a_{1}^{2}-a_{0}^{2}\right) \sin 2 m x_{0} \sin 2 k t}{\operatorname{sh}^{2} m h}\right] \\
& +\frac{m a_{1} a_{2}}{\operatorname{th} m h}\left[\cos 2 h t-\frac{\cos 2 m x_{n}}{2 \operatorname{ch}^{2} m h}\right]
\end{aligned}
$$

\section{Clapotis parfait :}

$$
\begin{aligned}
& x=x_{0}+\frac{2 a}{\text { th } m h} \sin k t \cos m x_{0}-\frac{m a^{2}}{4 \operatorname{sh}^{2} m h}\left\{\frac{(2 c h 2 m h+1)}{\operatorname{sh}^{2} m h} \cos 2 k t \sin 2 m x_{0}\right. \\
& \left.-\sin 2 m x_{0}\left[\frac{-6 \operatorname{sh} 2 m h-4 m h+\operatorname{ch} 2 m h(6 m h-\operatorname{sh} 2 m h)}{\operatorname{sh}^{2} m h+2 m h}+t^{2} m h \operatorname{ch} 2 m h\right]\right\} \\
& y=-2 a \sin m x_{0} \sin k t-\frac{m t^{2}}{\operatorname{th} m h}\left[1+\cos 2 k t+\frac{\cos 2 m x_{i}}{2 \operatorname{sh}^{2} m h}+\frac{3}{2} \frac{\cos 2 k t \cos 2 m x_{n}}{\operatorname{sh}^{2} m h}\right]
\end{aligned}
$$

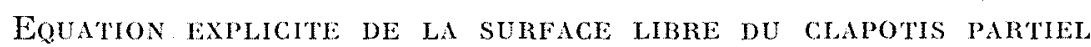

Les formules paramétriques (1) et (2) conviennent mal pour une étude détaillée de la surface libre; aussi avons-nous recherché une équation explicite de la surface libre.

Pour obtenir la cote vraie en $x_{0}$, à partir de la cote $y$ donnée par (2) au point $x$ et non $x_{0}$, nous devons rajouter à cette dernière cote l'expression $-\left(x-x_{0}\right) \frac{d y}{d x_{0}}$ qui fait intervenir la pente $\frac{d y}{d x_{0}}$ et l'écart $x-x_{10}$; nous avons ainsi :

$$
\begin{aligned}
x-x_{0}= & \frac{1}{\operatorname{th} m h}\left[\left(a_{1}-a_{0}\right) \sin k t \cos m x_{0}-\left(a_{1}-a_{2}\right) \cos k t \sin m x_{0}\right] \\
\frac{d ! y}{d x_{0}}= & -m\left[-\left(a_{1}+a_{2}\right) \sin m x_{0} \cos k t+\left(a_{1}+a_{2}\right) \cos m x_{0} \sin k t\right]-\left(x-x_{0}\right) \frac{d y_{0}}{d x_{0}} \\
= & \frac{m}{2 \operatorname{th} m h}\left[a_{1}^{2}+a_{2}^{2}+\left(a_{1}^{2}+a_{2}^{2}\right) \cos 2 m x_{0} \cos 2 k t\right. \\
& \left.\quad+2 a_{1} a_{2}\left(-\cos 2 k t+\cos 2 m x_{0}\right)-\left(a_{1}{ }^{2}-a_{2}{ }^{2}\right) \sin 2 m x_{0}^{\prime} \sin 2 k t\right]
\end{aligned}
$$

et nous obtenons ainsi l'écquation explicite de la surface libre $y$ en fonction de $x_{0}$ que nous pouvons rebaptiser $x$ sans inconvénient : 


$$
\begin{aligned}
& y=-\left[\left(a_{1}-a_{2}\right) \cos m x \cos k t+\left(a_{1}+a_{2}\right) \sin m x \sin k t\right]+\frac{m a_{1} a_{2}}{2 t h m h}(1+\operatorname{th} 2 m h) \cos 2 m x \\
& -\frac{m}{2 \operatorname{th} m h}\left(1+\frac{3}{2 \operatorname{sh}^{2} m h}\right)\left[\left(a_{1}^{2}+a_{2}^{2}\right) \cos 2 k t \cos 2 m x+\left(a_{1}^{2}-a_{2}^{2}\right) \sin 2 k t \sin 2 m x\right.
\end{aligned}
$$

L'équation (3) est, comme les équations (1) et (2), le début d'un développement en série de puissance de $a_{1}$ et $a_{2}$, mais nous croyons que la série (3) établie à partir des séries (1) et (2) est moins convergente que celle-ci. En tous les cas, du point de vue mathématique, l'équation (3) et les équations (1) et (2) ne sont pas équivalentes, mais ne doivent pas différer de termes d'ordre inférieur au troisième. Ces considérations interviennent surtout lorsque la cambrure devient importante ef qu'il y a lieu de discuter la validité des équations précédentes.

\section{MAXIMUM DE TA SURFACE LIBRE :}

Le but de cette étude étant essentiellement de pouvoir, à partir de mesures expérimentales, déterminer les amplitudes $a_{1}$ et $a_{2}$ d'un clapotis partiel de période et de longueur d'onde déterminées, il nous faut tout d'abord déterminer en quels points ces mesures devront être faites, c'est-àdire pour quelles valeurs de $m x$. La méthode classique consiste à faire deux séries de mesures, une aux ventres et une aux nœuds d'amplitude verticale. Ces nœuds et ventres sont définis par la propriété que l'amplitude verticale $y$ est respectivement minimum et maximum; on sait que si l'on se limite aux théories du premier ordre ils sont définis par $m x=(\pi / 2) \pm k \pi$ pour les ventres et $m x=0(-1 k \pi)$ pour les nœuds. Avec les théories du second ordre, on constate que les nœuds et les ventres répondant à la définition ci-dessus sont donnés par des valeurs de $m x$ olus complexes, si bien que l'on a intérêt à abandonner la notion classique de noud et de ventre, et à placer d'autorité les points de mesure en $m x=0(=k \pi)$ et $m x=(\pi / 2)+k \pi$. Le repérage des points de la dernière famille est rendu physiquement possible par le fait, facile à démontrer, qu'ils correspondent à la position maximum maximorum d'altitude (et non d'amplitude) atteinte par le profil. Leur repérage à la pointe est donc en principe facile. Les points de la série $m x=0( \pm k \pi)$ se déduisent des précédents par des translations de L/4.

ETUDE DE LA VARIATION DE NIVEAU EN $m x=(\pi / 2)(1+2 k)$.

Nous commencerons donc par étudier la variation de niveau en $m x=(\pi / 2)(1+2 k)$ en fonction du temps. Nous remarquerons que nous obtiendrons le même résultat en $m x=-(\pi / 2)(1+2 k)$ avec un déphasage dans le temps de $k t=\pi$.

Nous avons :

$$
y=-\left(a_{1}+a_{2}\right) \sin k t+\frac{m}{2 \operatorname{th} m h}\left(1+\frac{3}{2 \operatorname{sh}^{2} m h}\right)\left(a_{1}^{2}+a_{2}{ }^{2}\right) \cos 2 k t-\frac{m a_{1} a_{2}}{2 \operatorname{th} m h}(1+\operatorname{th} 2 m h)
$$

Les maxima et minima sont alors donnés par :

$$
y^{\prime}=0=-\left(a_{1}+a_{2}\right) \cos k t-\frac{2 m}{\operatorname{th} m h}\left(1+\frac{3}{2 \operatorname{sh}^{2} m h}\right)\left(a_{1}^{2}+a_{2}^{2}\right) \sin k t \cos k t
$$

qui s’annule pour :

$$
\begin{gathered}
k t=\frac{3 \pi}{2} \quad k t=\frac{\pi}{2} \\
\text { et } \sin k t=-\frac{\left(a_{1}+a_{2}\right)}{\frac{2 m}{\text { th } m h}\left(1+\frac{3}{2 \operatorname{sh}^{2} m h}\right)\left(a_{1}{ }^{2}+a_{2}{ }^{2}\right)}=\mathrm{N}
\end{gathered}
$$

Ces derniers extremums existent ou non suivant les valeurs des parametres. 
On peut montrer que lorsqu'ils existent, ce sont des maxima de $y$ (e'est-à-dire des points bas) et qu'ils sont supérieur's aux maxima relatifs donnés par lit $=(3 \pi / 2)$.

Posons:

$$
\beta=\frac{a_{2}}{a_{1}} \quad \frac{1}{\alpha}=\frac{2 \pi}{\operatorname{th} m h}\left(1+\frac{3}{2 \operatorname{sh}^{2} m h}\right) \text { (fig. 1) } \quad \alpha_{1}=\frac{a_{1}}{\alpha \mathrm{L}}
$$

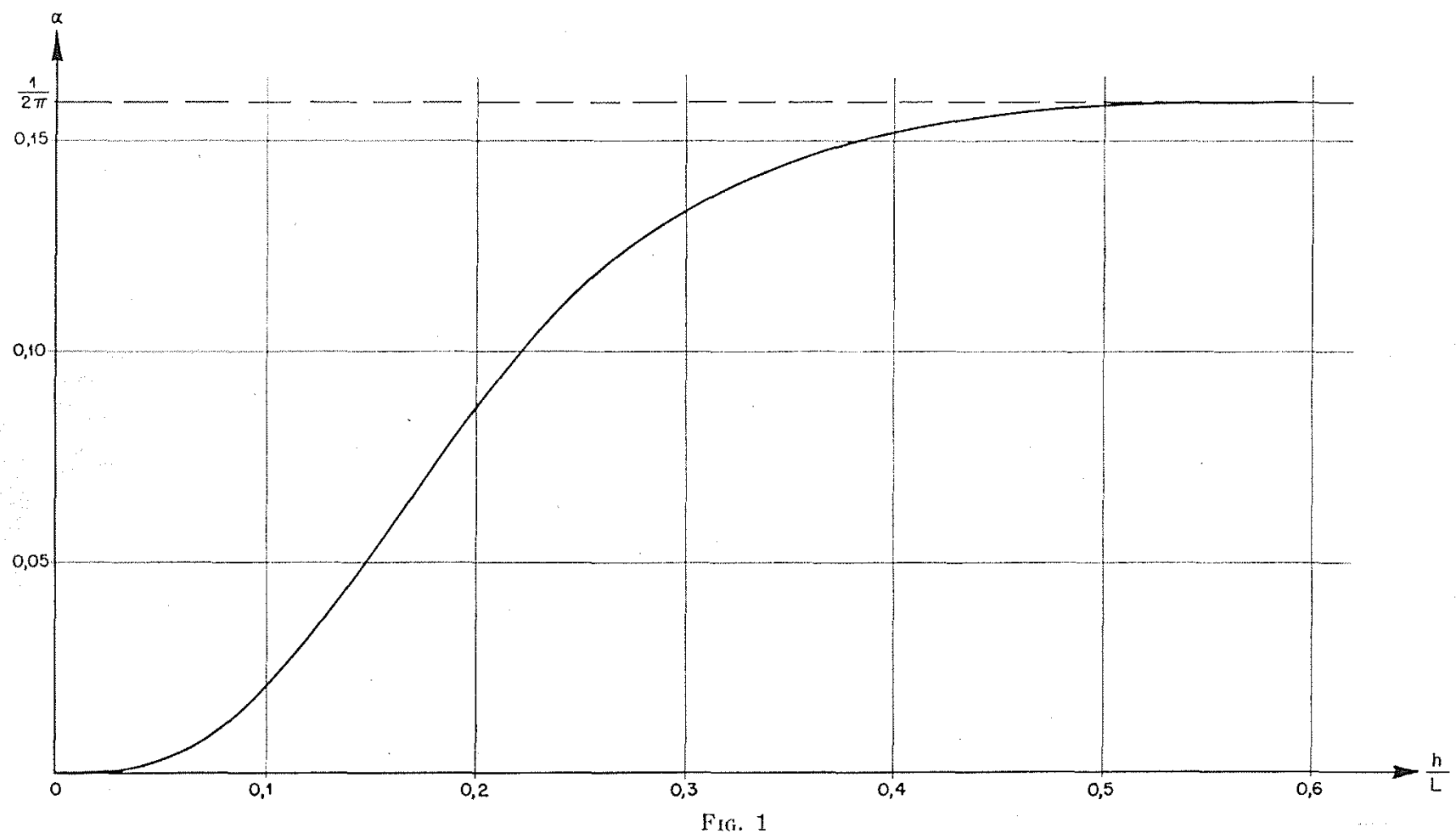

Nous pouvons tracer dans un plan $a_{1}, \beta$ une courbe de délinitation de l'existence de sinus qui est :

$$
\left.\alpha_{1}=\frac{1+\beta}{1+\beta^{2}} \quad \text { (fig. } 2\right)
$$

Les maxima et minima de $y$ sont alors les suivants :

$$
\begin{aligned}
& y_{\min }\left(k t=\frac{\pi}{2}\right)=\mathrm{M}_{2}=-\left(a_{1}+a_{2}\right)-\frac{m}{2 \operatorname{th} m h}\left(1+\frac{3}{2 \operatorname{sh}^{2} m h}\right)\left(a_{1}{ }^{2}+a_{2}{ }^{2}\right)-\frac{m a_{1} a_{2}}{2 \operatorname{th} m h}\left(1+\mathrm{th}^{2} m h\right) \\
& y_{\max }\left(k t=\frac{3 \pi}{2}\right)=\mathrm{M}_{1}=a_{1}+a_{2}-\frac{m}{2 \text { th } m h}\left(1+\frac{3}{2 \mathrm{sh}^{2} m h}\right)\left(a_{1}{ }^{2}+a_{2}{ }^{2}\right)-\frac{m a_{1} a_{1}}{2 \operatorname{th} m h}\left(1+\operatorname{th}^{2} m h\right) \quad \text { si } \mathrm{N}>1 \\
& y_{\mathrm{mil} . \mathrm{s}}=\mathrm{M}_{3}=-\left(a_{1}+a_{2}\right) \mathrm{N}+\frac{m}{2 \mathrm{th} m h}\left(1+\frac{3}{2 \mathrm{sh}^{2} m h}\right)\left(a_{1}{ }^{2}+a_{2}{ }^{2}\right)\left(1-2 \mathrm{~N}^{2}\right)-\frac{m a_{1} a_{10}}{2 \mathrm{th} m h}\left(1+\mathrm{hh}^{2} m h\right) \\
& \text { si } N<1
\end{aligned}
$$

Si nous appelons A la variation de niveau à $m x=(\pi / 2)$, nous avons :

$$
\mathrm{A}=\left|y_{\mathrm{max}}-y_{\min }\right|
$$

Soit :

$$
\left.\mathrm{A}=2\left(a_{1}+a_{2}\right)\right) \quad \text { si } \mathrm{N}>1
$$


et :

$$
\mathrm{A}=\left(a_{1}+a_{2}\right)(1-\mathrm{N})+\frac{m}{\operatorname{th} m h}\left(1+\frac{3}{2 \operatorname{sh}^{2} m h}\right)\left(a_{1}^{2}+a_{2}^{2}\right)\left(1-\mathrm{N}^{2}\right) \quad \text { si } \mathrm{N}<1
$$

ce qui donne, en remplaçant $\mathrm{N}$ par sa valeur :

$$
A=2\left(a_{1}+a_{2}\right)+\frac{\left[a_{1}+a_{2}-\left.\frac{2 m}{\operatorname{th} m h}\left(a_{1}{ }^{2}+a_{2}^{2}\right)\left(1+\frac{3}{2 \mathrm{sh}^{2} m h}\right)\right|^{2}\right.}{\frac{4 m}{\operatorname{th} m h}\left(a_{1}{ }^{2}+a_{2}^{2}\right)\left(1+\frac{3}{2 \operatorname{sh}^{2} m h}\right)}
$$

ETUde de la variation de NIVEaU len $m x=k \pi$.

Remarquons, comme précédemment, que l'on aurait aussi bien pu étudier la variation de niveau en $m x=\pi$.

Nous avons, pour $m x=0$ :

$$
y=-\left(a_{1}-a_{2}\right) \cos k t-\frac{m}{\operatorname{th} m h}\left(1+\frac{3}{2 \operatorname{sh}^{2} m h}\right)\left(a_{1}^{2}+a_{2}^{2}\right) \cos 2 k t+\frac{m a_{1} a_{1}}{2 \operatorname{th} m h}\left(1+\operatorname{th}^{2} m h\right)
$$

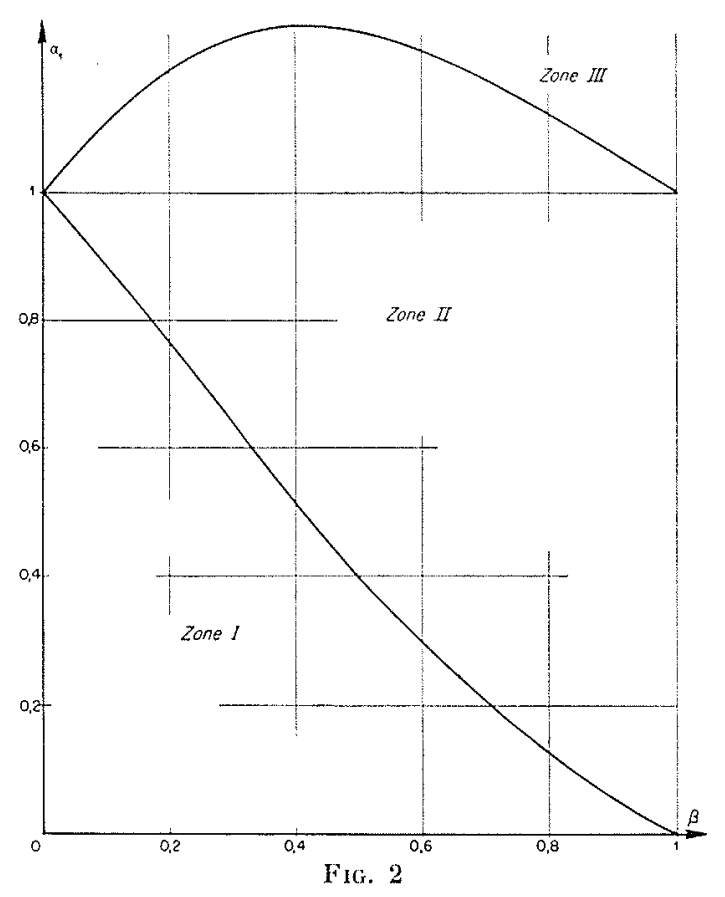

Les maxima et minima ont lieu pour $y^{\prime}=0$, c'est-à-dire :

$$
\begin{aligned}
& k t=0, \quad k t=\pi \text { et } \cos k t \\
& =-\frac{a_{1}-a_{0}}{\frac{2 m}{\operatorname{th} m h}\left(1+\frac{3}{2 \operatorname{sh}^{2} m h}\right)\left(a_{1}{ }^{2}+a_{2}{ }^{2}\right)}=\mathrm{P}
\end{aligned}
$$

Comme précédemment, la courbe d'existence du cosinus peut ètre tracée dans le plan $\alpha_{1}, \beta$; elle a pour équation :

$$
\alpha_{1}=\frac{1-\beta}{1+\beta^{2}}(\text { fig. 2) }) .
$$

Les maxima et minima de $y$ sont alors les suivants :

$$
\begin{aligned}
& y_{\operatorname{mix}}(k t=0)=\mathrm{M}_{6}=-\left(a_{1}-a_{2}\right)-\frac{m}{2 \operatorname{th} m h}\left(1+\frac{3}{2 \operatorname{sh}^{2} m h}\right)\left(a_{1}^{2}+a_{2}^{2}\right)+\frac{m a_{1} \cdot a_{1 .}}{2 \operatorname{th} m h}\left(1+\mathrm{th}^{2} m h\right) \\
& y_{\min }(k t=\pi)=\mathrm{M}_{5}^{\prime}=\left(a_{1}-a_{2}\right)-\frac{m}{2 \operatorname{th} m h}\left(1+\frac{3}{2 \operatorname{sh}^{2} m h}\right)\left(a_{1}^{2}+a_{2}^{2}\right)+\frac{m a_{1} a_{0}}{2 \operatorname{th} m h}\left(1+\mathrm{th}^{2} m h\right) \quad \text { si } \mathrm{P}>1 \\
& y_{\min }(\cos k t=\mathrm{P})=\mathrm{M}_{4}=-\left(a_{1}-a_{2}\right) \mathrm{P}+\frac{m a_{1} a_{2}}{2 \operatorname{th} m h}\left(1+\mathrm{th}^{2} m h\right)
\end{aligned}
$$$$
-\frac{m}{2 \operatorname{th} m h}\left(1+\frac{3}{2 \operatorname{sh}^{2} m h}\right)\left(a_{1}^{2}+a_{2}^{2}\right)\left(2 \mathrm{P}^{2}-1\right) \quad \text { si } \mathrm{P}<1
$$ 
Si on appelle $\mathrm{B}$ la variation de niveau à $m x=0$, nous avons :

$$
\begin{aligned}
& \mathrm{B}=\left|y_{\max }-y_{\min }\right| \\
& \mathrm{B}=2\left(a_{1}-a_{2}\right) \quad \text { si } \mathrm{P}>1 \\
& \mathrm{~B}=\left(a_{1}-a_{2}\right)(1-\mathrm{P})+\frac{m}{\mathrm{hh} m h}\left(1+\frac{3}{2 \mathrm{sh}^{2} m h}\right)\left(a_{1}{ }^{2}+a_{2}{ }^{2}\right)\left(\mathrm{P}^{2}-1\right) \quad \text { si } \mathrm{P}<1
\end{aligned}
$$

Ou en remplaçant $P$ par sa valeur :

$$
\mathrm{B}=2\left(a_{1}-a_{2}\right)+\frac{\left[a_{1}-a_{2}-\frac{2 m}{\operatorname{th} m h}\left(a_{1}{ }^{2}+a_{2}{ }^{2}\right)\left(1+\frac{3}{2 \operatorname{sh}^{2} m h}\right)\right.}{\frac{4 m}{\operatorname{th} m h}\left(a_{1}{ }^{2}+a_{2}{ }^{2}\right)\left(1+\frac{3}{2 \mathrm{sh}^{2} m h}\right)}
$$

\section{A B A Q UES}

Nous pouvons maintenant construire des abaques donnant, à partir des mesures expérimentales A et B, l'amplitude $a_{1}$ et l'amplitude $a_{2}$ ou le coefficient de réflexion $\beta=a_{2} / a_{1}$; pour ceci nous nous servirons des formules 5,6 , 8 et 9 donnant $\mathrm{A}$ et $\mathrm{B}$ en fonction de $a_{1}$ et $a_{2}$.

En examinant le plan $\alpha_{1}, \beta$, nous déterminons trois groupes de formules suivant que nous sommes de part et d'autre des courbes définies par (4) et (7) délimitant l'existence des sinus et des cosinus, définies précédemment. Nous appellerons ces divers cas zone I, II et III.

Pour pouvoir représenter les résultats par une seule famille de courbes, nous poserons :

$$
\alpha_{1}=\frac{a_{1}}{\alpha \mathrm{L}} \quad \alpha_{2}=\frac{a_{*}}{\alpha_{L}}
$$

et les formules précédentes deviennent après transformation, pour la :

\section{ZONE I}

$$
\begin{aligned}
& \frac{A}{\alpha \mathrm{L}}=\alpha_{1}+\alpha_{2}=\alpha_{1}(1+\beta) \\
& \frac{B}{\alpha \cdot L}=\alpha_{1}-\alpha_{2}=\alpha_{1}(1-\beta)
\end{aligned}
$$

\section{ZONE II}

$$
\begin{aligned}
\frac{A}{\alpha \mathrm{L}} & =\alpha_{1}+\alpha_{2}=\alpha_{1}(1+\beta) \\
\frac{\mathrm{B}}{\alpha \mathrm{L}} & =\alpha_{1}-\alpha_{2}+\frac{\left[\alpha_{1}-\alpha_{2}-\left(\alpha_{1}^{2}+\alpha_{2}^{2}\right)\right]^{2}}{4\left(\alpha_{1}{ }^{2}+\alpha_{2}^{2}\right)^{2}} \\
& =\alpha_{1}(1-\beta)+\frac{\left[1-\beta-\alpha_{1}\left(1+\beta^{2}\right)\right]^{2}}{4\left(1+\beta^{2}\right)}
\end{aligned}
$$

\section{ZONE III}

$$
\begin{aligned}
\frac{\mathrm{A}}{\alpha \mathrm{L}} & =\alpha_{1}+\alpha_{22}+\frac{\left[\alpha_{1}+\alpha_{0}-\left(\alpha_{1}{ }^{2}+\alpha_{0}{ }^{2}\right)\right]^{2}}{4\left(\alpha_{1}{ }^{2}+\beta^{2}\right)} \\
& =\alpha_{1}(1+\beta)+\frac{\left[1+\beta-\alpha_{1}\left(1+\beta^{2}\right)\right]^{2}}{4\left(1+\beta^{2}\right)} \\
\frac{\mathrm{B}}{\alpha \mathrm{L}} & =\alpha_{1}-\alpha_{22}+\frac{\left[\alpha_{1}-\alpha_{0}-\left(\alpha_{1}{ }^{2}+\alpha_{2}{ }^{2}\right)\right]^{2}}{4\left(\alpha_{1}{ }^{2}+\alpha_{2}{ }^{2}\right)} \\
& =\alpha_{1}(1-\beta)+\frac{\left[1-\beta-\alpha_{1}\left(1+\beta^{2}\right)\right]^{2}}{4\left(1+\beta^{2}\right)}
\end{aligned}
$$

Nous pensons que la manière la plus utile pour représenter abaques les formules précédentes est de porter :

en abscisse :

$$
\mathrm{D}=\frac{\mathrm{A}}{2 \approx \mathrm{L}}+\frac{\mathrm{B}}{2 \approx \mathrm{L}}
$$

et en ordonnée :

$$
R=\frac{A-B}{A+B}
$$

Nous avons alors pour les différentes zones les formules suivantes, qui ont été mises en abaques dans la figure 3 :

\section{ZONE I}

$$
\begin{aligned}
& \mathrm{D}=\alpha_{1} \\
& \mathrm{R}=\frac{\alpha_{2}}{\alpha_{1}}=\beta
\end{aligned}
$$




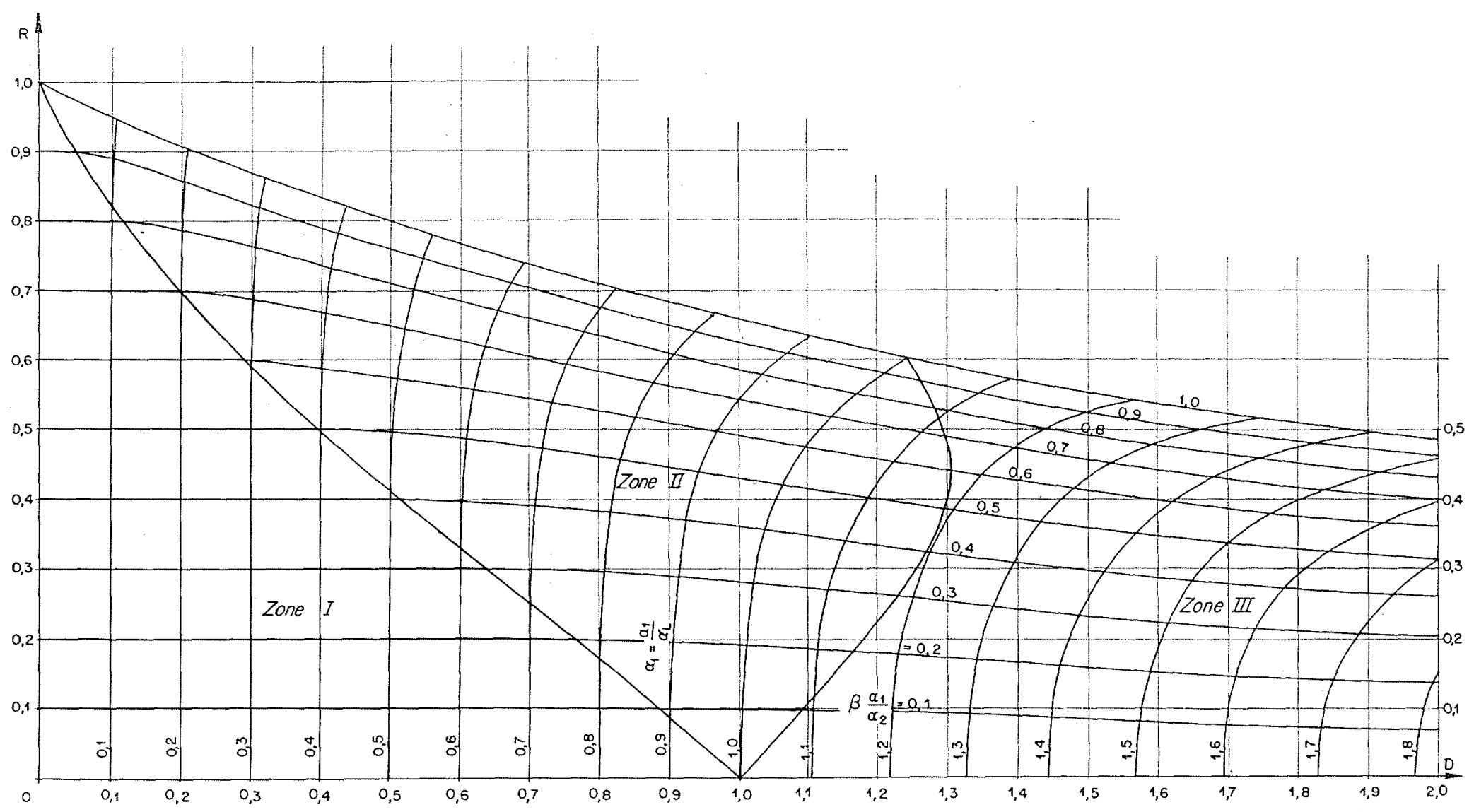

of

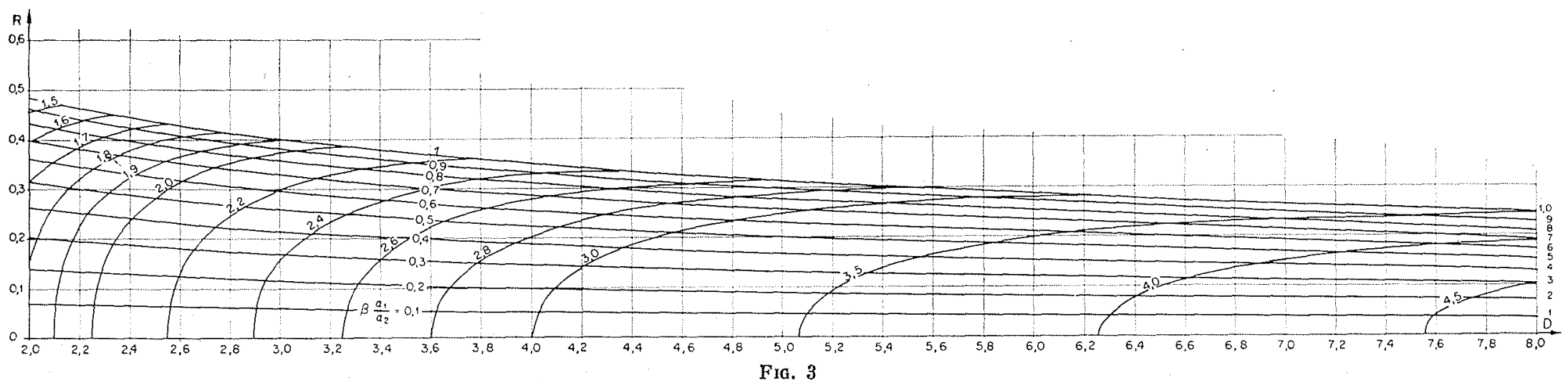


ZONE II

$$
\begin{aligned}
& \mathrm{D}=\alpha_{1}+\frac{\left.1-\beta-\alpha_{1}\left(1+\beta^{2}\right)\right]^{2}}{8\left(1+\beta^{2}\right)} \\
& \mathrm{R}=\frac{2 \alpha_{1} \beta-\frac{\left.1-\beta-\alpha_{1}\left(1+\beta^{2}\right)\right]^{2}}{4\left(1+\beta^{2}\right)}}{2 \alpha_{1}+\frac{\left[1-\beta-\alpha_{1}\left(1+\beta^{2}\right)\right]^{2}}{4\left(1+\beta^{2}\right)}}
\end{aligned}
$$

\section{ZONE III}

$$
\begin{aligned}
& \mathrm{D}=\alpha_{1}+\frac{1}{4}\left[\left(1-\alpha_{1}\right)^{2}+\alpha_{1}{ }^{2} \beta^{2}\right] \\
& R=\frac{\beta\left(\alpha_{1}+\frac{1}{1+\beta^{2}}\right)}{2 \alpha_{1}+\frac{1}{2}\left[\left(1-\alpha_{1}\right)^{2}+\alpha_{1}^{2} \beta^{2}\right]}
\end{aligned}
$$

Les courbes délimitant les zones s'écrivent alors :

zone I, zone II :

$$
\mathrm{D}=\frac{1-\mathrm{R}}{1+\mathrm{R}^{2}}
$$

zone II, zone III :

$$
\mathrm{I})=\frac{2+2 \beta+\beta^{2}}{2\left(1+\beta^{2}\right)} \mathrm{R}=\frac{\beta(2+\beta)}{2+2 \beta+\beta^{2}}
$$
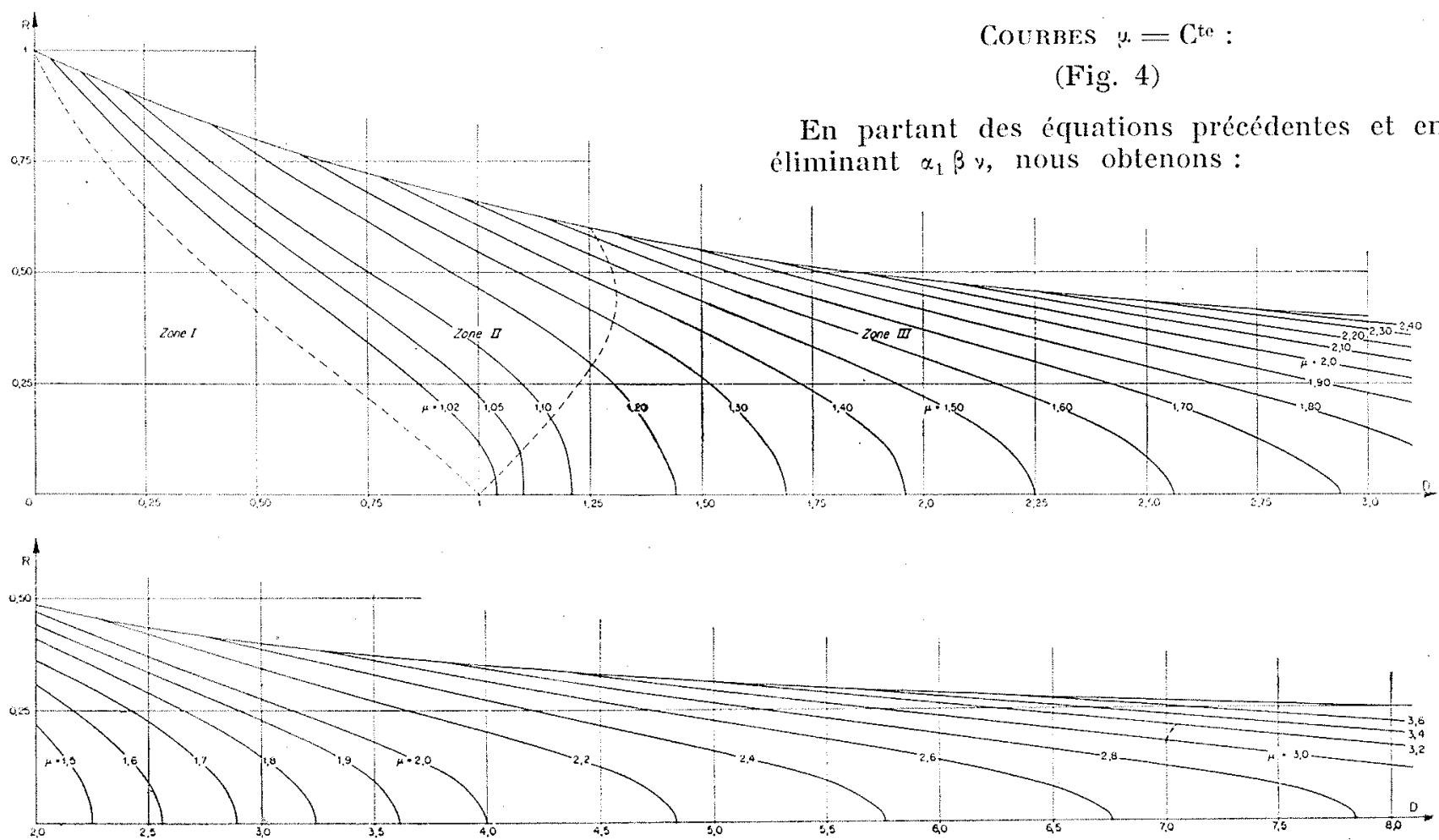

Fig. 4
Nous remarquerons d'autre part que la courbe $\beta=1$ est dans la zone II :

$$
\mathrm{D}=\frac{8(1-\mathrm{R})}{(1+\mathrm{R})^{2}}
$$

et dans la zone III :

$$
\mathrm{R}=\frac{1}{2 \mathrm{D}} \sqrt{2 \mathrm{D}-\frac{1}{4}}
$$

Nous constatons que les formules applicables à la zone I sont les mêmes que celles bien connues obtenues en ne tenant compte que des termes de premier ordre.

\section{*}

De facon à rendre plus précise la lecture du graphique, il nous a alors paru utile de tracer dans les zones II et III non les courbes $\alpha_{1}=\mathrm{C}^{\text {te }}$ et $\beta=\mathrm{C}^{\text {te }}$, mais des courbes donnant les valeurs relatives:

$$
\nu=\frac{\alpha_{1}}{\mathrm{D}} \quad \text { et } \quad y=\frac{\beta_{1}}{\mathrm{R}}
$$

En effet, on obtient ainsi directement la valeur des coefficients de correction qui sont voisins de l'unité dans la région où l'on utilisera le plus les abaques, c'est-à-dire principalement dans la zone II.

Dans la zone I, nous avons évidemment :

$$
\begin{gathered}
\mu=1 \quad y=1 \\
\text { Covnes } y=\mathrm{C}^{\mathrm{te}}:
\end{gathered}
$$

(Fig. 4)

En partant des équations précédentes et en éliminant $\alpha_{1} \beta \nu$, nous obtenons : 
ZONE II

$$
\begin{aligned}
\mathrm{D} & \left.=\frac{\mathrm{R} \mu+1}{(\mathrm{R}+1)\left(\mathrm{R}^{2} \mu^{2}+1\right)}\right\} 1-\mathrm{R} \mu+\frac{4 \mathrm{R}(\mu-1)}{\mathrm{R}+1} \\
& +\sqrt{\frac{8(\mu-1) \mathrm{R}}{\mathrm{R}+1}\left[\frac{2(\mu-1) \mathrm{R}}{1+\mathrm{R}}+1-\mathrm{R} \mu\right]}
\end{aligned}
$$

\section{ZoNe III}

$$
D=\frac{\mu^{2}\left[1+\sqrt{1-} R^{2}\right]}{2\left(1+R^{2} \mu^{2}\right)}
$$

où nous avons évidemment $\mu>1$

$$
\text { Courbes } v=\mathrm{C}^{\text {te }} \text { : }
$$

Si au lieu d'éliminer v, nous éliminons «., nous obtenons :

\section{ZONE II}

$$
\mathrm{D}=\frac{3-\mathrm{R}-2 \nu \pm \sqrt{8(1-\mathrm{R})(1-\nu)}}{2 \nu^{2}-2 \nu(\mathrm{R}+1)+(\mathrm{R}+1)^{2}}
$$

\section{ZoNe III}

$$
\mathrm{R}=\frac{\mathrm{D}(4-v)-1}{2 \mathrm{D}[2 \mathrm{D}(2-v)-1]} \sqrt{-\mathrm{D}^{2} v^{2}+2 \mathrm{D}(2-\nu)-1}
$$

avec évidemment : $\vee<1$.

Nous nous sommes limités à $\mathrm{D}<8$ pour les courbes précédentes en supposant que pour D $>8$ nous aurions des cambrures et des longueurs relatives trop importantes mettant en cause la validité des équations utilisées.

\section{CONCLUSION ET MODE D'EMPLOI DES ABAQUES.}

En résumé, nous avons établi les formules et les abaques au deuxième ordre près permettant, à partir de mesures expérimentales, de calculer les amplitudes de deux houles régulières de mème fréquence et de sens de propagation opposće, autrement dit du clapotis partiel.

A partir de la mesure de la profondeur d'eau au repos $h$, de la longueur d'onde $L$ nous calcuIons tout d'abord le paramètre sans dimension \% donnée soit par la figure 1, soit par la formule 3 bis. Ensuite, à partir des mesures de A, différence d'altitude entre crête et creux pris au point d'altitude maximum, et de $B$, différence d'altitude entre erête et creux pris à un point distant de $L / 4$ du point précédent, nous formons les quantités : ,

$$
\mathrm{D}=\frac{\mathrm{A}}{2 \alpha \mathrm{L}}+\frac{\mathrm{B}}{2 \alpha \mathrm{L}} \quad \text { et } \quad \mathrm{R}=\frac{\mathrm{A}-\mathrm{B}}{\mathrm{A}+\mathrm{B}}
$$

Nous pouvons alors soit nous servir de la figure 3, soit de la figure 4. Avec la figure 3, nous lisons $\beta=\left(a_{1} / a_{2}\right)$ et $\alpha_{1}=\left(\alpha_{1} / \alpha \mathrm{L}\right)$ où $\alpha_{1}$ et $\alpha_{2}$ sont les amplitudes recherchees des deux houles incidentes ou réfléchies. Avec la figure 4, nous lisons le nombre y. qui nous permet d'avoir $a_{1} / a_{0}=\beta$ par la relation $\beta=\mu \mathrm{R}$.

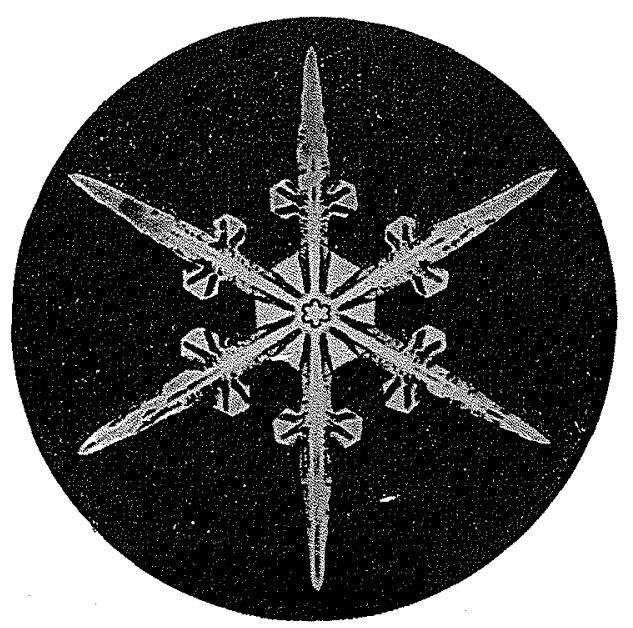

